\title{
Propuesta de manejo sustentable de Araucaria araucana (Mol. C. Koch)
}

\author{
Sustainable Propousal Management of Araucaria araucana (Mol. C. Koch)
}

\section{FERNANDO DRAKE A. ${ }^{1}$, MIGUEL ANGEL HERRERA ${ }^{2}$, EDUARDO ACUÑA C. ${ }^{1}$}

${ }^{1}$ Departamento Manejo de Bosques y Medioambiente, Facultad de Ciencias Forestales, Universidad de Concepción, Casilla 160 -C, Correo 3, Concepción, Chile. E-mail: fdrake@udec.cl ${ }^{2}$ ETS. Ingenieros Agrónomos y Montes, Departamento de Ingeniería Forestal, Universidad de Córdoba, Avda. Menéndez Pidal s/n, 14071 Córdoba, España.

\begin{abstract}
SUMMARY
The response of an Araucaria araucana forest, located at Cunco County, Ninth Region of Chile, was studied by applying a method that determines the cut allowable through the marginal analysis of volume and diameter class movement, with the aim of regulating forest. Following the method, a minimum diameter for harvesting of 110 $\mathrm{cm}$ and a harvest cycle of 37 years was established as suitable for ensuring the resource sustainability, preserving the forest capital, and keeping the structure and floral composition unchanged. Mediation may be considered using selective, sanitary, and silvicultural harvesting.
\end{abstract}

Keywords: Araucaria araucana, forest regulation, forest rotation.

\section{RESUMEN}

Fue estudiado el comportamiento de un bosque de Araucaria auraucana, localizado en la comuna de Cunco, IX Región de la Araucanía, Chile, mediante la aplicación de un método que determina la posibilidad de corta a través del análisis marginal del volumen y movimiento de clases diamétricas, con el objetivo de regular la masa boscosa. De acuerdo a este método se estableció un diámetro mínimo de corta igual a $110 \mathrm{~cm}$ y un ciclo de corta de 37 años, que asegura la sustentabilidad del recurso, conservando el capital vuelo, manteniendo inalterada la estructura y composición florística, mediante el uso de cortas selectivas, sanitarias y silvícolas.

Palabras clave: Araucaria auraucana, regulación forestal, ciclo de corta.

\section{INTRODUCCION}

El tipo forestal araucaria cubre una superficie de 253.715 ha, de las cuales 122.678 ha se encuentran incorporadas en el Servicio Nacional de Áreas Silvestres Protegidas del Estado (1). La especie Araucaria araucana (Mol. C. Koch), se presenta en la Cordillera de los Andes (2, 3, 4), cuya altura sobre el nivel del mar fluctúa entre los 900 y los 1.700 metros (5). En la Cordillera de Nahuelbuta, entre los 1.000 a 1.400 metros sobre el nivel del mar $(6,7)$.

Dentro de este tipo forestal existen diversas asociaciones, de acuerdo a la altitud y latitud en que se encuentren estos bosques. Se asocia con coigüe (Nothofagus dombeyi (Mirb.) Oerst.) y ocasionalmente con roble (Nothofagus obliqua (Mirb.) Oerst.var.) en los rangos inferiores de su distribución; con coigüe y lenga (Nothofagus pumilio (Poepp. et Endel.) Krasser.), en altitud intermedia; y con lenga en las superiores, hasta el límite de la vegetación arbórea. La asociación araucarialenga es la más característica del nivel altitudinal superior y la asociación araucaria-coigüe lo es en los sectores medios de las laderas occidentales de la Cordillera de los Andes, donde existe mayor precipitación $(8,9,10)$.

El clima en que se desarrolla la especie es templado cálido, de hielo y seco de estepa. El primero de ellos prevalece en la Cordillera de 
Nahuelbuta y además en el rango de distribución de bajas y medias altitudes en la Cordillera de los Andes. El clima de hielo, por efecto de altu$\mathrm{ra}$, es aquel propio de las mayores altitudes de la Cordillera de los Andes, donde escasos ejemplares y bosquetes de A. araucana crecen tanto hacia el sector chileno como al argentino $(11,12,13)$.

Generalmente, los bosques de A. araucana se encuentran degradados $y$, por lo tanto, para manejar este recurso es necesario hacer una transformación silvícola, permitiéndoles recuperarse y manejarlos de tal forma que vuelvan a tener una estructura semejante a la natural (14). Las intervenciones, para aprovechar esta especie en forma racional, deben ser tales que aseguren la formación de un estrato de regeneración. En éste debe existir una alta potencialidad, dada por la cantidad de semillas, la capacidad germinativa y una cobertura apropiada, y que debido a esto, probablemente, sea imprescindible intervenir la especie asociada $(15,16,17,18,19)$.

Los antecedentes que existen acerca de los posibles métodos silvícolas, a aplicar en $A$. araucana, señalan que esta especie debería ser manejada mediante el método de regeneración: selección o cortas sucesivas, en donde la araucaria fuese la especie principal. Debido a que la semilla de ésta es pesada, y por ello con dispersión a corta distancia $(20,21)$. En estudios realizados en relación a la estructura de distintos bosques vírgenes y explotados de araucaria, se explica la dinámica del desarrollo natural de estos bosques y el efecto que ha tenido la explotación sobre la regeneración. Según el desarrollo natural no habrían limitantes biológicas serias para manejar la araucaria. Si bien el crecimiento de la especie es extremadamente lento, en diámetro y altura, es favorable en cuanto a crecimiento como masa. El potencial productivo de los árboles y del bosque radica en los árboles de gran área basal; debido a estas características de crecimiento, razones de estabilidad del suelo, es aconsejable una forma de manejo que signifique mantener una cobertura permanente a través del manejo de un monte alto y regular con ciclos de corta (14). Para el manejo como monte alto irregular, se propone el método, de selección $(4,22,23,24,25)$, mediante ciclos de corta entre 20 y 40 años, para alcanzar edades que van desde los 300 a los 500 años $(23,25)$.

El modelo del bosque heteroetáneo equilibrado propone llevar la masa boscosa a una estructura multietánea con extracciones cíclicas en el tiempo, a través de un ciclo de corta determinado en forma matemática, en que se acumula el incremento que es capaz de formar el bosque en pie sin deteriorar su estructura y composición florística $(26,27)$. Este manejo permite modificar la duración de las fases de crecimiento, especialmente envejecimiento y desmoronamiento; y en lo posible eliminar esta última fase de modo que se aproveche el máximo de madera y evitando la pudrición por sobremadurez.

La legislación vigente entre los años 19871990, Decreto Supremo 141 (28), especificaba que la explotación de A. araucana, sólo podría efectuarse mediante el método selectivo o entresaca pie a pie, lo que se explicitó en una reglamentación y disposición técnica publicada en el Diario Oficial el 26 de diciembre de 1987.

De acuerdo a este decreto, el manejo de la $A$. araucana sería a partir de un diámetro mínimo de corta, bajo el cual no se permitían cortas productivas o de cosecha. Para su determinación, éste se define de acuerdo a la forma y el momento en que se realiza la corta, en aquellas clases de manejo, donde la variable edad y clase de edad carece de importancia o no es determinada; pero sí donde es relevante el concepto de ciclo de corta, que es el tiempo necesario para que sobre el capital vuelo del bosque irregular, se acumule un crecimiento susceptible de ser cortado de acuerdo con los objetivos del manejo.

Donoso (29) midió el efecto de intervenciones silvícolas, determinando que la explotación de $A$. araucana mediante el método de selección es adecuada, concluyendo que la intensidad de la intervención debe ser mayor que la permitida por la legislación vigente. Concluyendo que, con extracciones tan leves, el bosque en su conjunto no registra cambios de importancia en las tasas de crecimiento.

El presente estudio entrega un criterio de regulación en A. auraucana para mantener su composición florística, estructura y dinámica de la regeneración natural. Determina un diámetro mínimo y el ciclo de corta que permitiría mantener la sustentabilidad del recurso, aplicando el modelo de manejo de bosque heteroetáneo equilibrado.

\section{MATERIAL Y METODOS}

El estudio fue realizado durante el año 1988, en el predio "El Mirador", ubicado en la comuna 
de Cunco, IX Región de la Araucanía, Chile, en 258,9 ha cubiertas con el tipo forestal Araucaria - Coigüe. El estrato inferior de esta asociación estaba compuesto por quila (Chusquea quila (Mol.) Kunth.), canelillo (Drimys winteri var andina), rasca la choica (Desfontainea espinosa), Azara lanceolata, Berberis pearcei, Chillotrichium rosmarinifolium, Maitenus disticha y algunas plantas herbáceas como: Valeriana lapatipholia, que son propias en toda la Cordillera de los Andes. La regeneración natural es escasa dada la abundancia del sotobosque presente y el alto valor de cobertura.

El predio posee una topografía ondulada alta a quebrada, con unidad geomorfológica de plano remanente. El material no transportado y la superficie rocosa son del tipo volcánico andesítico-basáltico, presentando descomposición in situ. El suelo es profundo, con textura superficial franca, buen drenaje, estructura granular fina, desarrollo de perfil y estado de intemperización moderado (24).

La pendiente media del predio es de $16,2 \%$, dentro de la unidad de manejo existe un sector con pendiente mayor a $80 \%$ en una superficie de 0,6 ha. La altitud sobre el nivel del mar para el rodal oscila entre los 1.200 y $1.400 \mathrm{~m}$. La altura máxima del predio es de $1.518 \mathrm{~m}$ en su parte más elevada (1.430 m dentro del rodal) y $1.160 \mathrm{~m}$ en la parte más baja.

Para determinar la distribución y fases de desarrollo del bosque de A. araucana, interpretado a través del cambio de estructura, se procedió a realizar un censo poblacional en toda la extensión del rodal; y con el fin de calificar y cuantificar las especies arbóreas acompañantes se efectuó un muestreo sistemático.

En el censo se midió a cada individuo el diámetro a la altura del pecho (DAP) y el número de trozas de 3,70 m de largo. Para estimar la calidad de los individuos, se consideró como troza para aserrío, aquellas que presentaban buena forma, sanas y cilíndricas y factibles de utilizar en la producción de chapas.

En el muestreo de las especies asociadas, fueron empleadas unidades muestrales rectangulares de $500 \mathrm{~m}^{2}$, las que se establecieron en transectos lineales, en dirección oeste-este a una equidistancia de $300 \mathrm{~m}$. En el centro de la parcela se estableció una subparcela circular de $50 \mathrm{~m}^{2}$ para cuantificar la regeneración.
Para seleccionar las ecuaciones de distribución diamétrica se ajustaron diversos modelos que relacionan frecuencia expresado en número de árboles por ha versus DAP, los que fueron contrastados con los descritos por Donoso (30), Donoso y Lara (31) y Husch et al., (32), utilizándose para tal efecto el programa estadístico SPLUS 2000. En la selección de los modelos se analizan el coeficiente de determinación, error estándar de estimación y la distribución de errores.

Determinación del crecimiento anual periódico: Del censo poblacional se eligieron cinco individuos por marca de clase diamétrica a los que se les determinó el DAP con corteza mediante huincha diamétrica, y el crecimiento radial a través de tarugos extraídos con taladro incremental, en cada marca de clase se promedió el diámetro y los incrementos de los ejemplares medidos. Con estos antecedentes se determinó el Incremento Periódico del DAP, utilizando la metodología sugerida por Husch et al. (32).

Determinación del diámetro mínimo de corta: Con la proyección de la tabla de rodal se confeccionó una tabla de rodal y existencia futura para el término del ciclo de corta, acotado en la variable tiempo en 40 años (prefijado por el Decreto $\mathrm{Su}$ premo 141 (28)). Se estimó una incorporación a la primera clase de DAP, igual a la que existe actualmente por considerar una masa que regenera en las mismas condiciones edafoclimáticas. Determinado el crecimiento medio del DAP se ajustó el modelo DAP/crecimiento anual, y con estos valores ajustados para cada marca de clase, se proyectó una tabla de rodal. Con el incremento periódico del diámetro, se determinó un factor de movimiento multiplicando el incremento por el ciclo de corta y dividido a su vez por la amplitud de la clase. El factor de movimiento indica el porcentaje de árboles de cada clase en cuestión que ascenderían de una a tres clases. Los decimales determinan el porcentaje que ascendería una clase, que corresponde a una clase más que la indicada por el primer dígito entero; la diferencia a ese porcentaje ascendería el número de clases indicada explícitamente por este dígito.

Para calcular el diámetro mínimo de corta se empleó del método basado en análisis marginal. Este método establece un diámetro de corta en que el diámetro mínimo de corta iguala el diáme- 
tro medio correspondiente al máximo volumen medio por centímetro de diámetro medio. Se procedió a su determinación mediante los siguientes niveles de análisis:

1. Determinación del volumen acumulado en base a la tabla de rodal y existencia presente.

2. Determinación del incremento medio del volumen medio por centímetro de diámetro medio.

3. Determinación del incremento periódico del volumen medio por centímetro de diámetro medio.

\section{RESULTADOS Y DISCUSION}

Estructura: La estructura del bosque es la de una población multietánea con presencia de varios estratos, en bosquetes en distintas etapas o fases de desarrollo, y con diferente proporción de las especies participantes descritas por Hillgarter (33). Esto determinó la diferenciación de ciclos de desarrollo continuos. Las formas y las secuencias de las fases se describen a continuación.

Fase de crecimiento óptimo: Esta ocupó un 15\% de la superficie, con especie dominante A. araucana, y en proporciones similares $N$. dombeyi y $N$. pumilio. Esta fase se caracterizó por la escasa regeneración, abundancia relativa de sotobosque y por la presencia de un bosque multiestratificado en altura, en edad y diámetro. La mayor participación está presente en individuos jóvenes, maduros y envejecidos y muy pocos en etapa de desmoronamiento, ratificado esto por escaso desprendimiento de placas en la parte basal de $A$. araucana, pudrición interna y ganchos secos en la parte superior.

Fase de envejecimiento: Aproximadamente en un $52 \%$ del rodal, se ubican individuos que ocupan un estrato superior, en etapa de senectud, sin presencia de regeneración, excepto en sectores muy pequeños y elevados en montículos donde el sotobosque es menos denso. Los árboles son de gran tamaño, con bajo crecimiento periódico diametral, dominando en altura A. araucana. La especie $N$. dombeyi está constituida por gran densidad de número de árboles por hectárea, cuyos individuos han ingresado a la fase de senectud y desmoronamiento; no obstante $N$. pumilio presenta una menor participación respecto a las especies anteriores.
Fase de envejecimiento y desmoronamiento. En la parte alta del predio, cubriendo una extensión de un $33 \%$ de la superficie del rodal, se combinan las fases de desmoronamiento y envejecimiento, encontrándose sectores con alta regeneración de $A$. araucana y $N$. pumilio, y escaso sotobosque.

La regeneración de $A$. araucana tiene una distribución agregada y heterogénea, observándose que la mayor parte de la superficie no presenta regeneración abundante dada la alta participación de $N$. pumilio. En aquellos lugares donde el canelillo y Desfontainea son escasos y disminuye la quila, la regeneración es mayor. Cabe destacar que en esta área $N$. dombeyi disminuye considerablemente su participación como especie acompañante.

Estado Sanitario: A. araucana presenta buena forma, la conicidad de los individuos es más frecuente en aquellos ejemplares que no han alcanzado la etapa de desmoronamiento y sobremadurez. No se constató ataque de hongos o insectos que descalifiquen la madera en individuos jóvenes y maduros.

$N$. dombeyi, tiene forma regular, con presencia de árboles bifurcados, fustes torcidos, baja altura comercial y de sección diametral ovalada. Los problemas sanitarios en esta especie están presentes en aquellos individuos en estado de sobremadurez, con aparición de ganchos de gran diámetro y área de copa sin actividad fotosintética, se observó pudrición central.

N. pumilio presentó individuos de baja calidad, tanto en la forma como en aspectos de sanidad. Debido a esto en las tablas de rodal y existencia no se registran volúmenes comerciales por su baja altura y fustes perforados, lo que descalifica la madera de esta especie.

Distribución diamétrica de las especies: Especie A. Araucana: Se midieron 24.991 ejemplares con DAP entre 10 y $195 \mathrm{~cm}$, rango diamétrico para el cual fue ajustado satisfactoriamente un modelo del tipo exponencial (figura 1), correspondiente a la estructura del bosque multietáneo. Schmidt et al. (14) en un estudio realizado para esta especie en el área de Lonquimay, reconoce la existencia de estructura multietánea homogénea, donde el cambio de estructura y regeneración son 


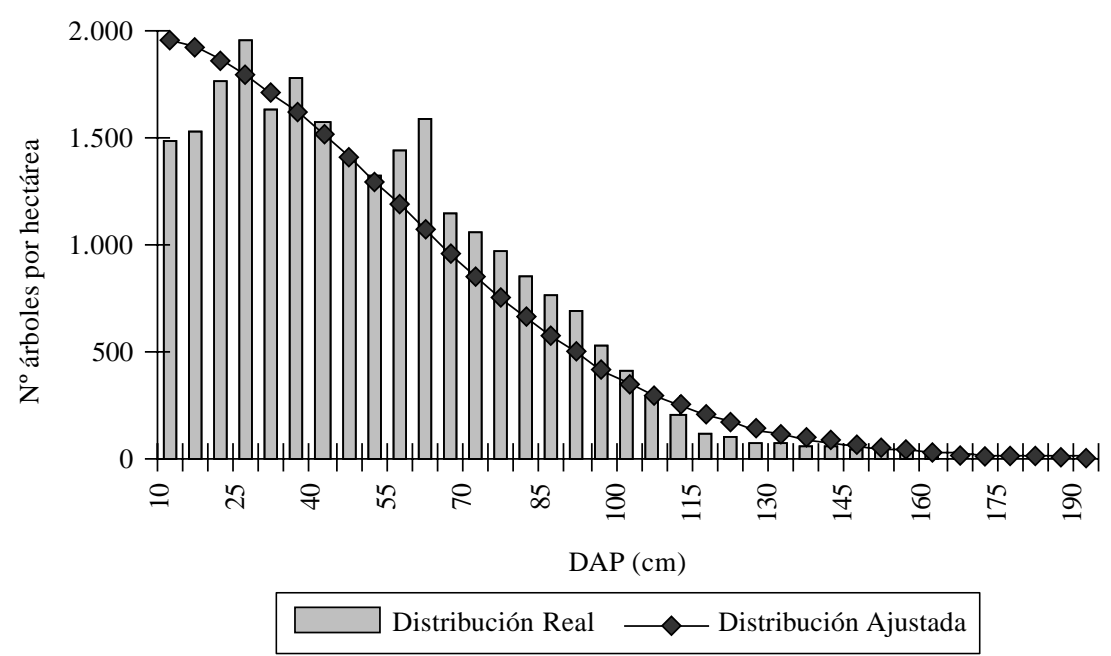

Figura 1. Distribución diamétrica real y ajustada de Araucaria araucana según modelo exponencial.

Real and fitted diametric distribution of Araucaria araucana based on an exponential model.

producto de la acción individual de los árboles en desmoronamiento.

Especies Acompañantes: Al observar la distribución y el modelo ajustado para coigüe y lenga (figuras 2 y 3), explica que la velocidad de crecimiento y longevidad son diferentes a la de $A$. araucana, revelando la existencia de varias generaciones para las nothofagaceas, ello determinará entonces variaciones o alteraciones para el establecimiento de regeneración de A. araucana.
La regeneración de todas las especies no se produce gradualmente en un dosel arbóreo que va muriendo lentamente (22), sino que ocurre discontinuamente bajo claros creados en el dosel. El proceso de regeneración es al azar y no puede ser predecible como un fenómeno cíclico, debido a las alteraciones alogénicas y la disponibilidad de semillas, que puedan producir plantas de las especies asociadas que accederán al sitio, dependiendo de los fenómenos de floración y de producción de semillas (21). En definitiva, el concepto de dinámica cíclica para bosque

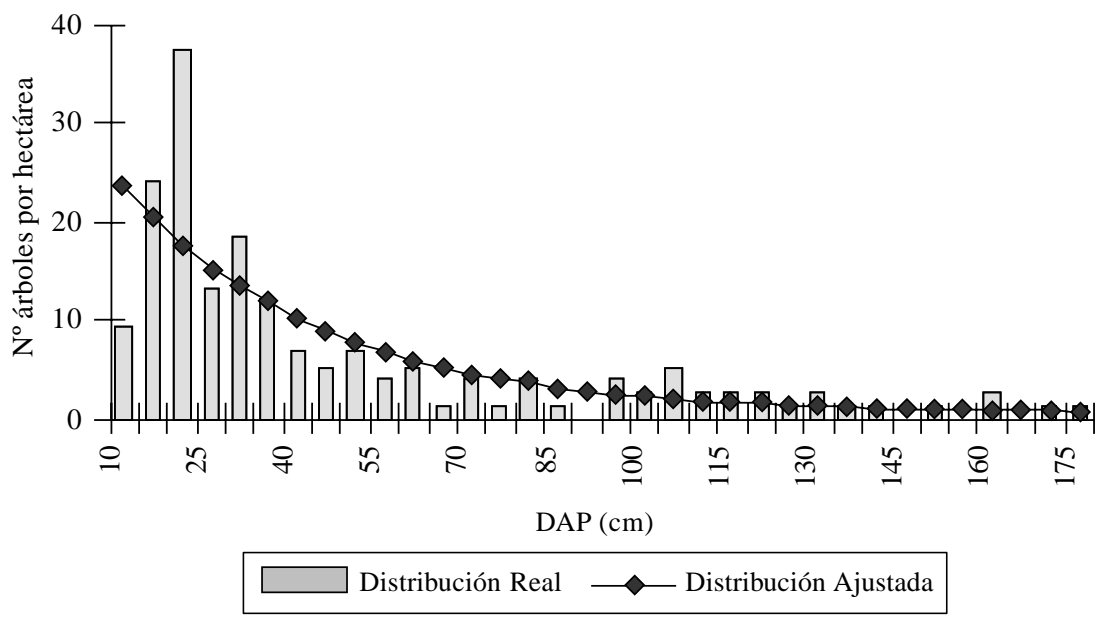

Figura 2. Distribución diamétrica real y ajustada de Nothofagus dombeyi según modelo exponencial.

Real and fitted diametric distribution of Nothofagus dombeyi based on an exponential model. 


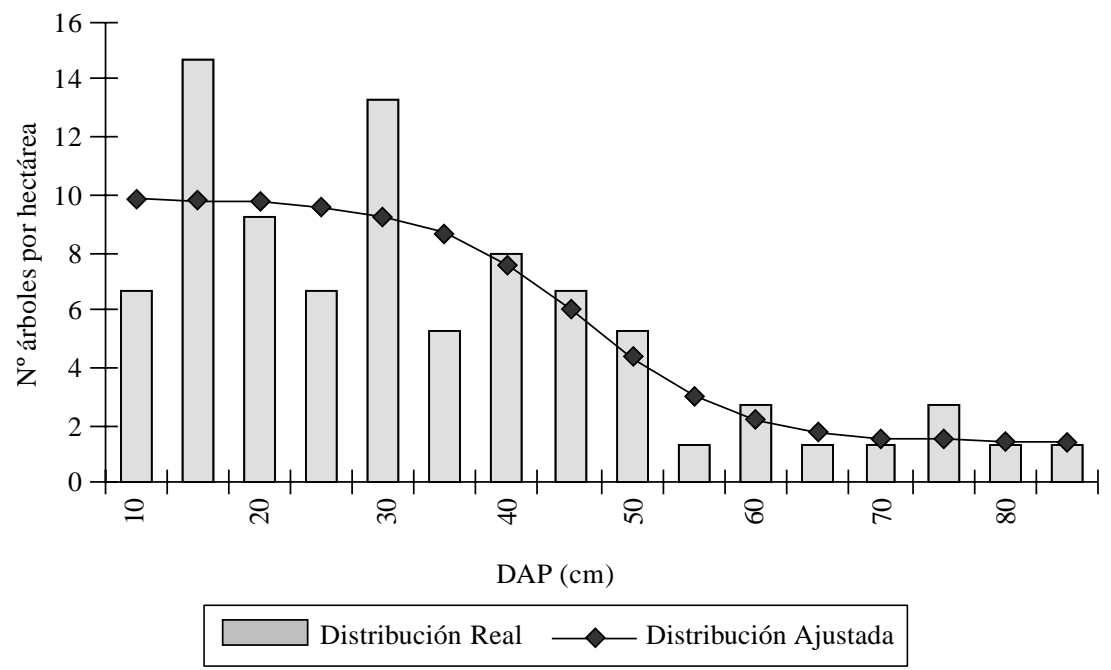

Figura 3. Distribución diamétrica real y ajustada de Nothofagus pumilio según modelo exponencial.

Real and fitted diametric distribution of Nothofagus pumilio based on an exponential model.

y más aún para bosques mixtos, se confunde con la regeneración por claros, estrategia que se produce a nivel de individuos que poseen ciclos de vida independiente, y no de rodales.

La dinámica regenerativa de los bosques de A. araucana y de las nothofagaceas es muy similar a la asociación $N$. pumilio- $N$. dombeyi, siendo este último un colonizador más agresivo, que en áreas alteradas por efectos catastróficos invade violentamente formando rodales coetáneos. Sin embargo, A. araucana, más tolerante, es capaz de establecerse en pequeña proporción en esos rodales coetáneos en la medida que ellos se abran por autorraleo, dejando pasar alguna luminosidad. En la medida que estos rodales maduran llegando a una etapa avanzada y equilibrio dinámico, la asociación es capaz de regenerar mediante estrategias de claros, siendo coigüe y lenga dependientes de claros mayores, por ser especies intolerantes (30).

Volumen: Con las funciones de volumen de $A$. araucana por troza (3,6 metros de longitud) para diferentes diámetros se determinó la existencia volumétrica (34).

De acuerdo a la tabla de rodal y existencia, $N$. dombeyi participa mayoritariamente en número de árboles y en área basal por hectárea, pero su volumen es inferior al registrado por A. araucana (cuadro 1), lo que se explica por la calidad infe-

\section{CUADRO 1}

Tabla de rodal y existencia para Araucaria araucana, Nothofagus dombeyi y Nothofagus pumilio.

Stand and stock table of Araucaria araucana, Nothofagus dombeyi and Nothofagus pumilio

\begin{tabular}{|l|cc|cc|cc|}
\cline { 2 - 7 } \multicolumn{1}{c|}{} & \multicolumn{2}{c|}{ Densidad } & \multicolumn{2}{c|}{ Área basal } & \multicolumn{2}{c|}{ Volumen } \\
\hline Especies & $\left(\mathrm{arb} \mathrm{ha}^{-1}\right)$ & $(\%)$ & $\left(\mathrm{m}^{2} \mathrm{ha}^{-1}\right)$ & $(\%)$ & $\left(\mathrm{m}^{3} \mathrm{ha}^{-1}\right)$ & $(\%)$ \\
\hline Araucaria araucana & 171,00 & 38,20 & 44,79 & 40,20 & 206,03 & 52,50 \\
Nothofagus dombeyi & 188,00 & 42,10 & 56,55 & 50,70 & 186,07 & 47,50 \\
Nothofagus pumilio & 88,00 & 19,70 & 10,13 & 9,10 & - & - \\
\hline
\end{tabular}


rior de los individuos, dada la descalificación de trozas producto de su mala forma y estado sanitario. En general, la distribución del rodal es agregada y heterogénea.

La distribución de volumen por clase diamétrica para $A$. araucana, dio como resultado un modelo ajustado de distribución normal (figura 4).

Ciclo de corta de Araucaria araucana: La fijación del cupo de corta, basado sólo en la determinación del incremento periódico del DAP, no relaciona la culminación del comportamiento del área basal, volumen y sus incrementos medios y periódicos, para establecer un análisis tridimensional de la masa boscosa (figura 5), no obstante es posible fijar un diámetro de liquidación del rodal a través del diámetro mínimo de corta en que este se iguala a la extracción del volumen acumulado, a partir de las clases diamétricas superiores prefijadas. Para llevar a cabo este análisis es necesario graficar el volumen acumulado por centímetro de diámetro medio y los incrementos medio y periódico por centímetro de diámetro medio, determinando el punto de intersección en que ambos incrementos logran la igualdad; es en este punto donde se ha prefijado el diámetro de liquidación. En este caso en particular, el diámetro mínimo de corta es igual a la clase diamétrica $110 \mathrm{~cm}$; a partir del límite superior de la clase se entra a cortar el bosque (figura 6).

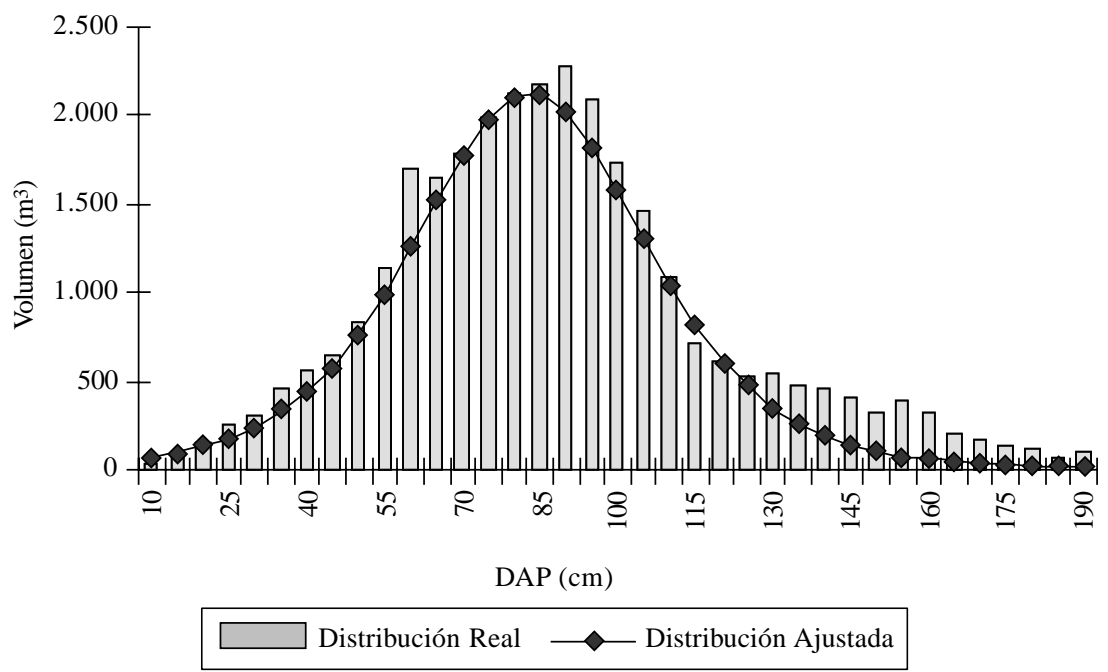

Figura 4. Distribución del volumen $\left(\mathrm{m}^{3}\right)$ Araucaria araucana versus DAP $(\mathrm{cm})$. Volumetric distribution $\left(\mathrm{m}^{3}\right)$ of Araucaria araucana versus DBH $(\mathrm{cm})$.

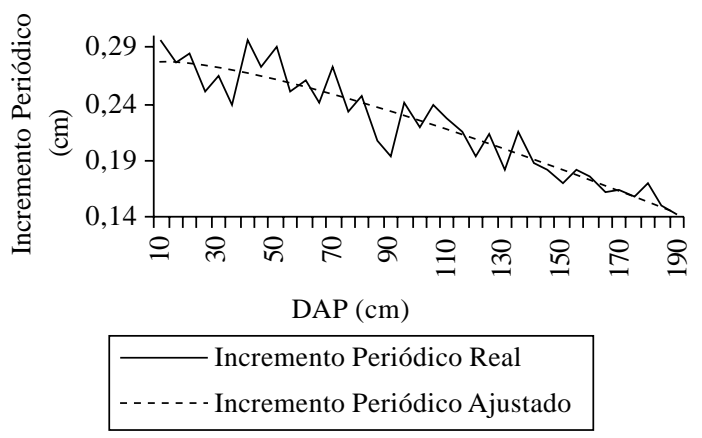

Figura 5. Incremento periódico real y ajustado (cm) para Araucaria araucana versus DAP $(\mathrm{cm})$.

Araucaria araucana real and fitted periodic increment $(\mathrm{cm})$ versus DBH $(\mathrm{cm})$. 


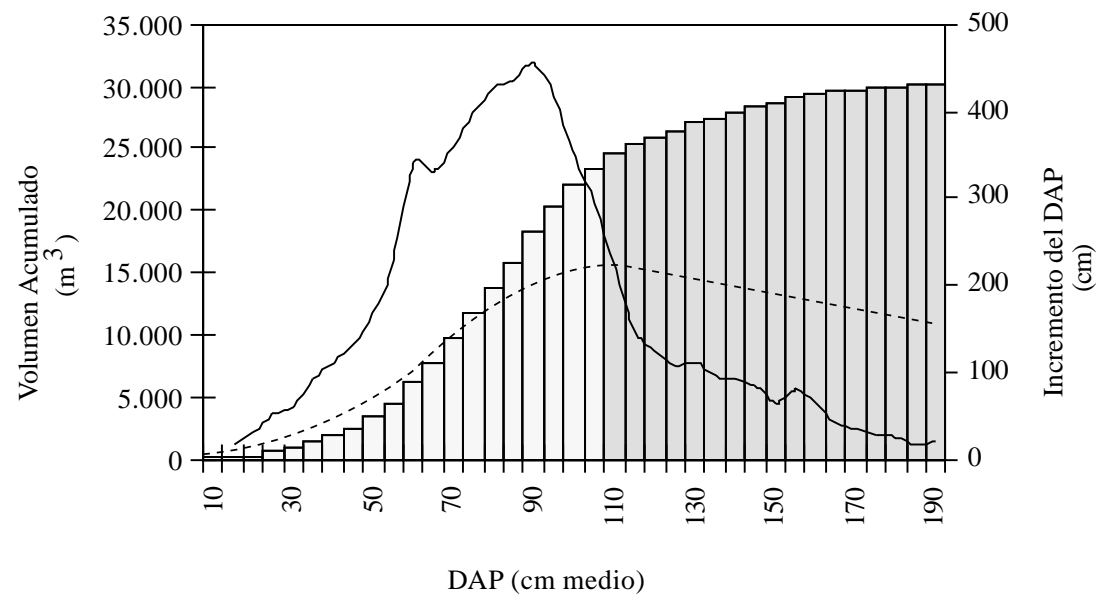

Volumen Acumulado

- Incremento Periódico

Incremento Medio

Figura 6. Volumen acumulado $\left(\mathrm{m}^{3}\right)$, incremento periódico y medio por centímetro de diámetro medio (punto de intersección que determina el diámetro de liquidación del bosque).

Accumulated volume $\left(\mathrm{m}^{3}\right)$, periodic and mean increment per $\mathrm{cm}$ of mean diameter (intersection point determines the forest liquidation).

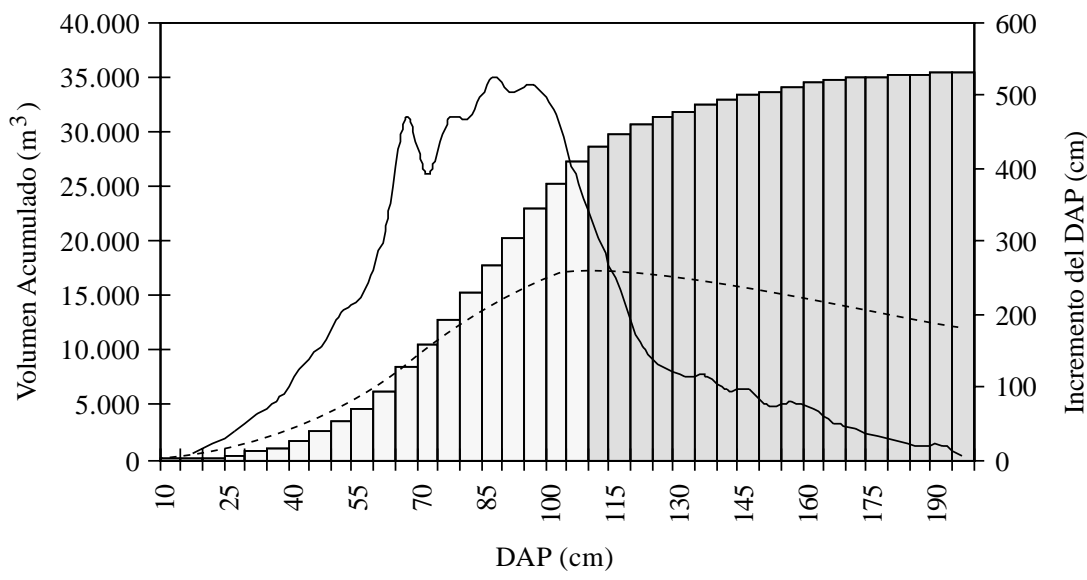

Volumen Acumulado Incremento Periódico Incremento Medio

Figura 7. Volumen acumulado e incrementos proyectados a 40 años. El diámetro de corta es superior al presente. Observe que este es de $115 \mathrm{~cm}$.

Accumulated volume $\left(\mathrm{m}^{3}\right)$ and projected increments in 40 years. The cut diameter is higher than the current value $(115 \mathrm{~cm})$.

$\mathrm{Al}$ analizar la figura 7 se puede visualizar que los incrementos medio $\left(223,43 \mathrm{~m}^{3} \mathrm{~cm}^{-1}\right.$ por centímetro de diámetro medio), y periódico $(218,75$ $\mathrm{m}^{3} \mathrm{~cm}^{-1}$ por centímetro de diámetro medio) se igualan en la clase diamétrica $110 \mathrm{~cm}$, Esto establece una posibilidad de corta de $6.719,898 \mathrm{~m}^{3}$, equivalente a 857 árboles, es decir, aproximadamente seis árboles por hectárea y 45,83 $\mathrm{m}^{3} \mathrm{ha}^{-1}$. Este volumen asumiendo el ciclo de corta establecido por el método de incremento diametral igual a 40 años da como resultado un incremento medio anual de $1,146 \mathrm{~m}^{3} \mathrm{ha}^{-1} \mathrm{año}^{-1}$. 
Al proyectar el crecimiento del bosque en 40 años, de acuerdo a los valores de incremento periódico en centímetros registrados en el tiempo presente, se generó un factor de movimiento de clases diamétricas que proyectó los valores futuros de las frecuencias, de tal forma que, al considerar que el ciclo de corta de 40 años y al aplicar el método de análisis marginal con estas nuevas frecuencias, se puede observar que la clase diamétrica que corresponde al punto en que se igualan los crecimientos medio y periódico, que al momento del inventario fijó un diámetro mínimo de corta igual a $110 \mathrm{~cm}, 40$ años más tarde, el movimiento de las clases de frecuencias determina que en el futuro la igualdad entre ambos incrementos fija un diámetro mínimo de corta igual a $115 \mathrm{~cm}$ (figura 7); esto significa que el capital vuelo reservado en este ciclo de corta establece un sobre stock de crecimiento, lo que lleva a concluir que para mantener el estado de crecimiento sostenido del bosque con un diámetro de corta igual a $110 \mathrm{~cm}$ debería considerar un ciclo de corta levemente inferior a 40 años, es decir, 26 años.

\section{CONCLUSIONES}

- El comportamiento analizado en bosque de $A$. Araucana - N. dombeyi - N. pumilio tras el censo poblacional, se determinó que el análisis marginal es un método que permite establecer diámetros mínimos de corta y que con el apoyo de la metodología de movimiento de clases diamétricas, es posible definir ciclos de corta que aseguran sostenibilidad y sustentabilidad del recurso.

- Se deben intervenir todas las clases sociales de la masa boscosa mediante marcación de los individuos en todo el rodal, permitiendo que en la selección estén presentes individuos que serán extraídos por corta selectiva, corta sanitaria y corta silvícola.

- Se deben generar funciones de volumen locales para precisar la metodología por su grado de dependencia en el cálculo de los incrementos corriente y medio, correspondiente a las tangente trigonométrica y geométrica del modelo de volumen acumulado en $\mathrm{m}^{3}$ por centímetro de diámetro medio.

- La determinación del diámetro de corta y ciclo de corta por análisis marginal y proyección futura de la frecuencia de los individuos por clase diamétrica, con fidelidad de funcio- nes de volúmenes aceptada, es un método apropiado para manejar bosques en cualquier tipo o asociación forestal.

\section{AGRADECIMIENTOS}

Los autores desean expresar con gratitud los agradecimientos al Ingeniero Forestal señor Fernando Drake Martín, por su disposición en el aporte de la revisión del trabajo y posterior presentación en el Segundo Congreso Chileno de las Ciencias Forestales llevado a cabo en la Universidad Austral de Chile, en noviembre de 2004 y al Ingeniero Forestal señor Marco Rubilar González por la colaboración en el procesamiento de la información.

\section{BIBLIOGRAFIA}

(1) CONAF, CONAMA, BIRF, Universidad Austral de Chile, Pontificia Universidad Católica de Chile and Universidad Católica de Temuco. Catastro y evaluación de los recursos vegetacionales nativos de Chile. Informe Nacional con Variables Ambientales. Santiago, Chile. 1999. 88 p.

(2) DONOSO, C. Tipos forestales de los bosques nativos de Chile. Documento de Trabajo $\mathrm{N}^{\circ} 38$. Investigación y Desarrollo Forestal FAO/DP/CHI/76/003. Santiago, 1981, 70 p.

(3) URZUA, A. STRUKTUR. Und Zuwachsuntersuchungen in magellanischen Nothofagus pumilio Urwáldrem als Grundlage eines Modells der waldbaulichen Uberfuhurungsplanung Diss., Universitat Göttingen, 1981, 128 p.

(4) CAVIERES, A. Estudio de crecimiento de Araucaria araucana (Mol.) C. Koch en un bosque virgen de Araucaria-Lenga. Tesis Ing. Forestal. Escuela de Ciencias Forestales, Universidad de Chile. 1987, 98 p.

(5) VEBLEN, T.T. Regeneration patterns in Araucaria araucana forests in Chile. J. of Biogeography, 1982, vol. 9, $\mathrm{N}^{\mathrm{o}}$ 1, p. 11-28.

(6) MONTALDO, P. La bio-ecología de la Araucaria araucana (Mol.) Koch. Instituto Forestal Latinoamericano de Investigación y Capacitación. Instituto Forestal Latino-Americano de Investigación y Capacitación. Boletín del Instituto Forestal Latino-Americano de Investigación y Capacitación, 1974, № 46-48, p. 03-55.

(7) MUÑOZ, M. Crecimiento de un bosque de Araucaria araucana (Mol.) Koch bajo distintas intensidades de corta. Tesis Facultad de Ciencias Forestales. Facultad de Ciencias Forestales, Universidad de Chile, 2000, 70 p.

(8) GAJARDO, R. Vegetación del bosque de Araucaria araucana (Mol.) Koch en la Cordillera de Los Andes (Lonquimay, provincia de Malleco). En: Boletín Técnico $\mathrm{N}^{\circ}$ 57, Facultad de Ciencias Forestales, Universidad de Chile. Santiago, 1980, 25 p.

(9) DONOSO, C. Ecología Forestal. El bosque y su medio ambiente. Editorial Universitaria, Santiago, Chile.1981, $369 \mathrm{p}$.

(10) VEBLEN, T., B. BURNS, T. KITZBERGER, A. LARA, R. VILLALBA. The ecology of conifers of Southern South America. In: Ecology of the southern conifers 
(Enright, N.J. and Hill, R.S., eds), Melbourne University Press, Carlton, Victoria. 1995, p. 120-155.

(11) MONTALDO, P. Condiciones ecológicas y dasonómicas de la especie Araucaria araucana (Mol.) Koch. Tesis de Facultad de Agronomía, Universidad de Chile, 1951, $135 \mathrm{p}$.

(12) MUJICA, R. Untersuchungen zur waldbaulichen Behandlung von Araucaria araucana Wäldern in Südchile. Dr. Thesis, Technische Universität München, Fakultät für Ernährung, Landnutzung und Umwelt, 2001, 202 p.

(13) BURNS, B.R. Regeneration dynamics of Araucaria araucana. Ph.D. Thesis, University of Boulder, University of Colorado, 1991, 211 p.

(14) SCHMIDT, H., M. TORAL, P. BURGOS. Aspectos de estructura y regeneración natural para el manejo silvícola de los bosques de Araucaria - Lenga en Chile. En: Problemas Forestales de Género Araucaria. Encuentro IUFRO. Curitiba, Brasil.1980, p. 159-166.

(15) PUENTE, M. Utilización de un bosque del tipo Araucaria con criterio de permanencia., Facultad de Ciencias Forestales, Universidad de Chile, 1980. Boletín Técnico $\mathrm{N}^{\circ} 57.22 \mathrm{p}$.

(16) MORALES, J. Estudio de crecimiento de Nothofagus pumilio (Lenga), en un bosque virgen de Araucaria araucana - Nothofagus pumilio (Araucaria-Lenga), en el sector de Lonquimay IX Región. Tesis Facultad de Ciencias Agrarias y Forestales. Escuela de Ciencias Forestales, Universidad de Chile, 1983, $81 \mathrm{p}$.

(17) DONOSO, C., V. GERDING, V. OLIVARES, P. REAL, V. SANDOVAL, R. SCHLATTER, F. SCHLEGEL. Antecedentes para el manejo del bosque nativo de Forestal Arauco (Sector Cordillera de Nahuelbuta). Informe de Convenio $\mathrm{N}^{\circ} 74$. Proyecto Forestal Arauco/U. Austral de Chile.1984, 79 p.

(18) DONOSO, S., H. SCHMIDT. Crecimiento de regeneración de Araucaria araucana (Mol.) Koch con diversas intervenciones silvícolas. Ciencias Forestales, 1991, vol. 7, $\mathrm{N}^{\mathrm{o}} 1 / 2$, p. 13-19.

(19) GONZÁLEZ, A. Análisis de la densidad y crecimiento de la regeneración en un bosque de Araucaria araucana bajo distintas intensidades de cortas de selección. Tesis Facultad de Ciencias Forestales, Universidad de Chile, 2001, 79 p.

(20) VITA, A. Los tratamientos silviculturales. $2^{\text {a }}$ ed. Universidad de Chile, Facultad de Ciencias Agrarias y Forestales, 1996, $156 \mathrm{p}$.
(21) MUÑOZ, R. Análisis de la productividad de semillas de Araucaria araucana (Mol.) C. Koch en el área de Lonquimay - IX Región. Tesis. Facultad de Ciencias Agrarias, Veterinarias y Forestales, Universidad de Chile, 1984, $93 \mathrm{p}$.

(22) SCHMIDT, H. Dinámica de un bosque virgen de Araucaria - Lenga (Chile). Bosque, 1977, vol. 2, $\mathrm{N}^{\circ}$ 1, p. 3-11.

(23) SCHMIDT, H., M. TORAL, P. BURGOS. Silvicultura y uso del bosque de Araucaria. Región Lonquimay. Facultad de Ciencias. Forestales, Universidad de Chile, 1977, 28 p.

(24) PERALTA, M. Geomorfología, clima y suelo del tipo forestal Araucaria en Lonquimay. Facultad de Ciencias Forestales, Universidad de Chile, 1980, Boletín Técnico $\mathrm{N}^{\circ} 57.53 \mathrm{p}$

(25) SCHMIDT, H., A. LARA. Descripción y potencialidad de los bosques nativos de Chile. Ambiente y Desarrollo, 1985 , vol. $1, \mathrm{~N}^{\mathrm{o}} 2$, p. $91-108$

(26) DAVIS, K.P. Forest management: Regulation and valuation. $2^{\text {nd }}$ ed. McGraw-Hill, New York, NY., 1966, $519 \mathrm{p}$.

(27) DAVIS, L., K. JOHNSON. Forest management. $3^{\text {nd }}$ ed. McGraw-Hill, New York, 1987, 790 p.

(28) DIARIO OFICIAL, Santiago, Chile. 23 de mayo de 1969, Decreto Supremo 157. 05 de diciembre de 1970, Decreto Supremo 439. 17 de febrero de 1975, Decreto Supremo 292. 26 de abril de 1976, Decreto Supremo 29. 26 de diciembre de 1987, Decreto Supremo 141.

(29) DONOSO, S. Crecimiento de Araucaria araucana (Mol.) Koch bajo diversas intensidades de corta. Tesis. Facultad de Ciencias Forestales, Universidad de Chile, 1990, $73 \mathrm{p}$.

(30) DONOSO, C. Bosques templados de Chile y Argentina. Variación estructura y dinámica Editorial Universitaria, Santiago, Chile, 1993, 484 p.

(31) DONOSO, C., A. LARA. Silvicultura de los bosques nativos de Chile. Editorial Universitaria. Santiago, Chile, 1998, $421 \mathrm{p}$.

(32) HUSCH, B., T.W. BEERS, J.A. KERSHAW. Forest mensuration. $4^{\text {th }}$ Edition. John Wiley and Sons, Inc. New Jersey. 2003, 443 p.

(33) HILGARTER, F.W. Waldbauliche und estragskundliche Untersuchungen im subalpinen Fichtenurwald Scatle/Brigels. Diss. Nr 4619. Univ. Zürich, 1971, 80 p.

(34) INFOR. Tabla de volumen por troza para araucaria (Araucaria araucana (Mol.) C. Koch). Instituto Forestal, Santiago, Chile. 1969, Nota Técnica No 13, 35 p. 